\title{
Simple integration of fast excitation and offset, delayed inhibition computes directional selectivity in Drosophila
}

\author{
Eyal Gruntman $\odot$, Sandro Romani $\odot$ and Michael B. Reiser $\oplus^{*}$
}

\begin{abstract}
A neuron that extracts directionally selective motion information from upstream signals lacking this selectivity must compare visual responses from spatially offset inputs. Distinguishing among prevailing algorithmic models for this computation requires measuring fast neuronal activity and inhibition. In the Drosophila melanogaster visual system, a fourth-order neuron-T4-is the first cell type in the ON pathway to exhibit directionally selective signals. Here we use in vivo whole-cell recordings of T4 to show that directional selectivity originates from simple integration of spatially offset fast excitatory and slow inhibitory inputs, resulting in a suppression of responses to the nonpreferred motion direction. We constructed a passive, conductance-based model of a T4 cell that accurately predicts the neuron's response to moving stimuli. These results connect the known circuit anatomy of the motion pathway to the algorithmic mechanism by which the direction of motion is computed.
\end{abstract}

T he computation of directional selectivity has been studied for decades in both vertebrate and invertebrate visual systems and has given rise to competing algorithmic models ${ }^{1}$. The Hassenstein-Reichardt (HR) detector uses a synergistic combination of offset excitatory inputs ${ }^{2}$ to enhance responses to motion in the preferred direction, whereas the Barlow-Levick (BL) detector uses inhibitory input to 'veto' an offset excitatory input ${ }^{3}$, suppressing motion in the nonpreferred, or null, direction.

The HR detector, which was originally formulated to account for motion detection in insects, has become a canonical example of a neuronal computation ${ }^{1,4}$. This model has endured because its elegant mechanism accounted for a wide array of behavioral results ${ }^{2,5,6}$ and for the detailed response properties of large motion-sensitive output neurons of the fly visual system ${ }^{1,7,8}$. Recent progress on the visual circuits upstream of these motion sensitive neurons in Drosophila has revealed that stimuli are processed through ON and OFF pathways ${ }^{9-14}$. In the ON pathway, the columnar T4 neurons are the first cell type to exhibit directionally selective signals ${ }^{10,11,14,15}$; four T4 subtypes are each directionally selective in one of four cardinal directions ${ }^{9,11,16}$.

A prevalent hypothesis for how T4 cells could implement the HR detector was proposed based on different input cell types providing the temporally ${ }^{14}$ and spatially ${ }^{9}$ offset inputs, but further analysis of the circuit ${ }^{15,17}$ has ruled out this circuit mechanism. The other recent approach to identifying the mechanism responsible for directional selectivity in the T4 circuit has been to use calcium imaging to measure T4 responses to structured visual stimuli. Several studies found evidence for enhanced responses to preferred-direction motion $^{18,19}$, while several others found both preferred-direction enhancement and null-direction suppression ${ }^{20-22}$. A number of groups have recently proposed that motion detection in flies may be implemented as a hybrid mechanism featuring elements of both the HR and BL algorithms $s^{15,21-23}$. However, direct evidence for either mechanism has been elusive, in large part because these studies rely on calcium imaging. Calcium indicator responses are insensitive to fast events, obscuring the small timing differences required by the HR detector, and are also insensitive to hyperpolarization, thereby preventing the direct measurements of inhibition, a defining feature of the BL model.

\section{Results}

To directly measure the physiological properties of T4 neurons, we used targeted in vivo whole-cell electrophysiology. We confirmed the identity of GFP-labeled T4 neurons by measuring reliable depolarizations in response to small ON flashes (Fig. 1a and Supplementary Fig. 1). We used online stimulus generation and analysis to localize the center (within $\sim 2^{\circ}$ ) of the receptive field (RF) of each recorded neuron (Supplementary Fig. 1b,c). To measure directional selectivity in single T4 cells, we presented a narrow $\left(\sim 2^{\circ}\right.$ wide) ON bar moving in eight different directions through the mapped RF center (Fig. 1b,c). The T4 membrane potential showed small depolarizations in response to several directions of motion (Fig. 1b) but large responses to movement in a $\sim 90^{\circ}$ range, centered around the preferred direction (PD; opposite to the null direction, $\mathrm{ND}$ ), in agreement with the tuning width measured with calcium imaging ${ }^{11}$. Our moving-bar stimulus generates apparent motion: the bar appears to move in a series of discrete $\left(\sim 2^{\circ}\right)$ steps, where the speed of movement is determined by the duration the bar remains at each position. To examine the computation of directional selectivity across a behaviorally relevant range, we presented four movement speeds. The responses to the moving stimuli were averaged across individual recordings once aligned to the PD (Fig. 1c). We quantified the strength of directional selectivity using a directional selectivity index (DSI, defined in Methods) and saw significant tuning at all stimulus speeds $(P<0.05$, one sided unpaired $t$ test $)$, with stronger directional selectivity at the two slower speeds $(P<0.05$, one sided paired $t$ test; Fig. 1d). At slow speeds, the membrane potential exhibited prominent hyperpolarizing responses, both following the response to PD motion and preceding the depolarizing response to ND motion (Fig. 1c; detailed in Supplementary Fig. 2). The sequence of depolarizing and hyperpolarizing signals depended on the direction of motion, suggesting that inhibitory and excitatory inputs to T4 may be spatially offset, in agreement with recent anatomical findings ${ }^{17}$. 


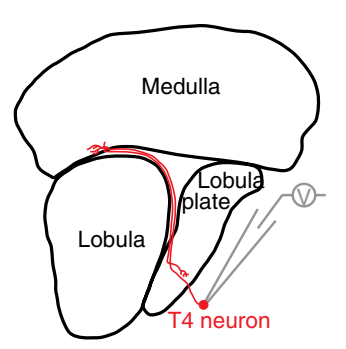

b Single T4 recording (cell 14) $28 \% \mathrm{~s}$

$\mathbf{k}$

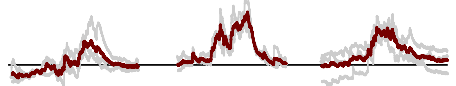

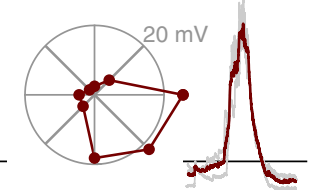

$28 \%$ moving-bar stimulus
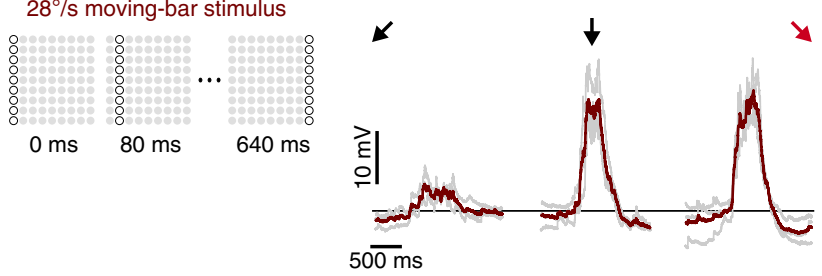

C Averaged aligned T4 recordings

d
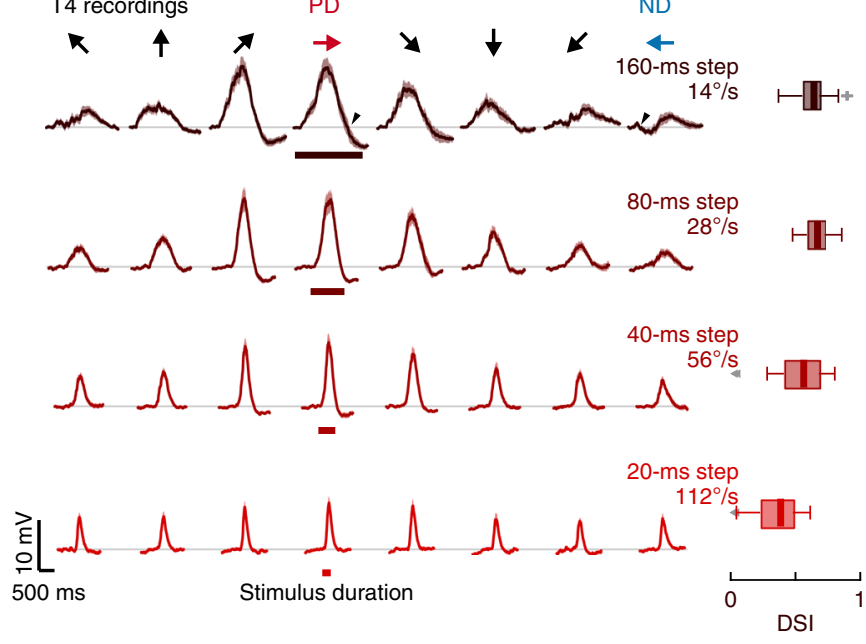

Fig. 1 | Whole-cell recordings of T4 cells show directionally selective responses. a, Top: schematic of Drosophila visual system with example T4 cell; whole-cell recordings are targeted to cell body. Bottom: a moving bar ( $1 \times 9$ LEDs) is presented as a sequence of discrete steps. $\mathbf{b}$, Responses to a bright bar moving in eight directions (indicated by arrows) at $28^{\circ} / \mathrm{s}$. Individual trial responses in gray ( $n=3$ trials); mean in maroon. Center: response to each direction at the time of maximal directional selectivity.

c, Baseline-subtracted responses ( $n=17$ cells) to bar motion, aligned to the PD of each cell (mean \pm s.e.m.). Arrows represent the direction of stimulus motion. Arrowheads indicate hyperpolarization following (or preceding) response to $\mathrm{PD}$ (or ND) motion. Colored horizontal bars indicate stimulus presentation. $\mathbf{d}$, DSI for bar motion responses ( $n=17$ cells). Crosses represent outliers, and triangles denote data points outside of the plot (see Methods for boxplot conventions).

Mapping the $\mathrm{T} 4$ receptive field reveals a spatiotemporal asymmetry. In order to characterize the fine structure of the T4 RF, we first localized the RF center and identified the PD-ND axis. We decomposed the moving-bar stimulus into its elementary components-single-position bar flashes with a fixed duration-and presented them at all positions, randomly interleaved, along the PD-ND axis. Aligning the single-position flash responses (SPFRs) based on the position of peak depolarization (Fig. 2a) revealed two key properties. (i) The center and leading side of the T4 RF exhibited excitatory responses.
These responses were larger and lasted longer for longer flash stimuli (corresponding to slower speeds). Additionally, longer flashes presented on the leading side of the RF revealed depolarizing responses that were not observed for short flashes. (ii) The inputs along this axis were spatially asymmetrical; hyperpolarizing inputs only appeared on the trailing side of the RF (most clearly seen for the longer duration flashes). Overlaying voltage traces from leading and trailing RF positions exposed a temporal asymmetry in the inputs (Fig. 2b,d). Responses on the trailing side decayed faster than responses at equivalent positions on the leading side. This temporal sharpening can be seen across speeds, including the shortest duration flash where hyperpolarization was not directly recorded (Fig. 2b).

For the longer-duration flash stimuli, our RF mapping (Fig. $2 \mathrm{c}$ and Supplementary Fig. 3) shows a spatially offset distribution of depolarizing and hyperpolarizing inputs to T4, with the hyperpolarizing input on the trailing side of the RF. Notably, the offset between the peaks of excitatory and inhibitory inputs $\left(\sim 6^{\circ}\right.$ of visual angle; Supplementary Fig. $3 \mathrm{~b}$ ) corresponds to the approximate angular separation between adjacent ommatidia on the fly eye, matching the predicted structure for the inputs of the Drosophila motion detector ${ }^{5}$. Because we did not measure excitatory and inhibitory currents directly, the underlying overlap between excitation and inhibition is expected to extend beyond positions where we can reliably measure hyperpolarizing input.

If T4 implements an HR-like mechanism, we expect that SPFRs on the leading side of the RF would be time-delayed relative to trailing-side SPFRs ${ }^{2,14}$. A simple expectation for relative time delays between offset visual stimulation generated through differential filtering by either upstream cells and/or synaptic transmission is that the responses would occur with a relative temporal offset. However, the onset time of the flash response was constant across the RF (Fig. 2d,e). Nevertheless, a significant position effect was seen in the decay time (Fig. 2d,e), which we attribute to temporal sharpening (Fig. 2b) due to overlapping inhibitory inputs on the trailing side of the RF (Fig. 2c). Rather than a relative time delay between offset inputs, we find that the temporal differences in the SPFRs can be explained by fast excitatory inputs on the leading side combined with slower inhibitory inputs on the trailing side of the RF.

Directional selectivity results from ND suppression. The classical models for motion detection use a nonlinear combination of offset inputs, resulting in either an enhancement of PD responses (HR) or a suppression of ND responses (BL). A simple test for directional selectivity is to deliver flash stimuli at two positions in the T4 RF and examine whether the response to sequential stimulation in one direction is larger than the response to the opposite sequence of stimulation. We implemented this two-step apparent-motion stimulus using single-position flashes ( $160 \mathrm{~ms}$ in duration) for pairs of positions around the RF center (Fig. 3a). To assess the effect of direction, we compared each two-step response to the superposition of the corresponding two single-position responses (offset in time and summed; Fig. 3b). This comparison revealed no enhanced response to the second bar appearing toward the PD but a reduction in the second bar response when it appears toward the ND. Flashes separated by $\sim 10^{\circ}$ (bottom row) did not appear to interact, showing neither enhancement nor suppression. Furthermore, the suppression was most prominent for bar pairs 'moving' in the ND that begin on the trailing side, consistent with our finding that the distribution of inhibitory inputs is skewed towards this side of the RF. By comparing the apparent-motion responses to the same bar pairs but in opposite directions (Fig. 3c), we found that the leading side of the RF-corresponding to the primarily excitatory domain of the SPFRs (Fig. 2)-was motion blind (see also Supplementary Fig. 4). Strong directional selectivity was only seen for the bar pairs that included the trailing side of the RF and was only produced by ND suppression. 


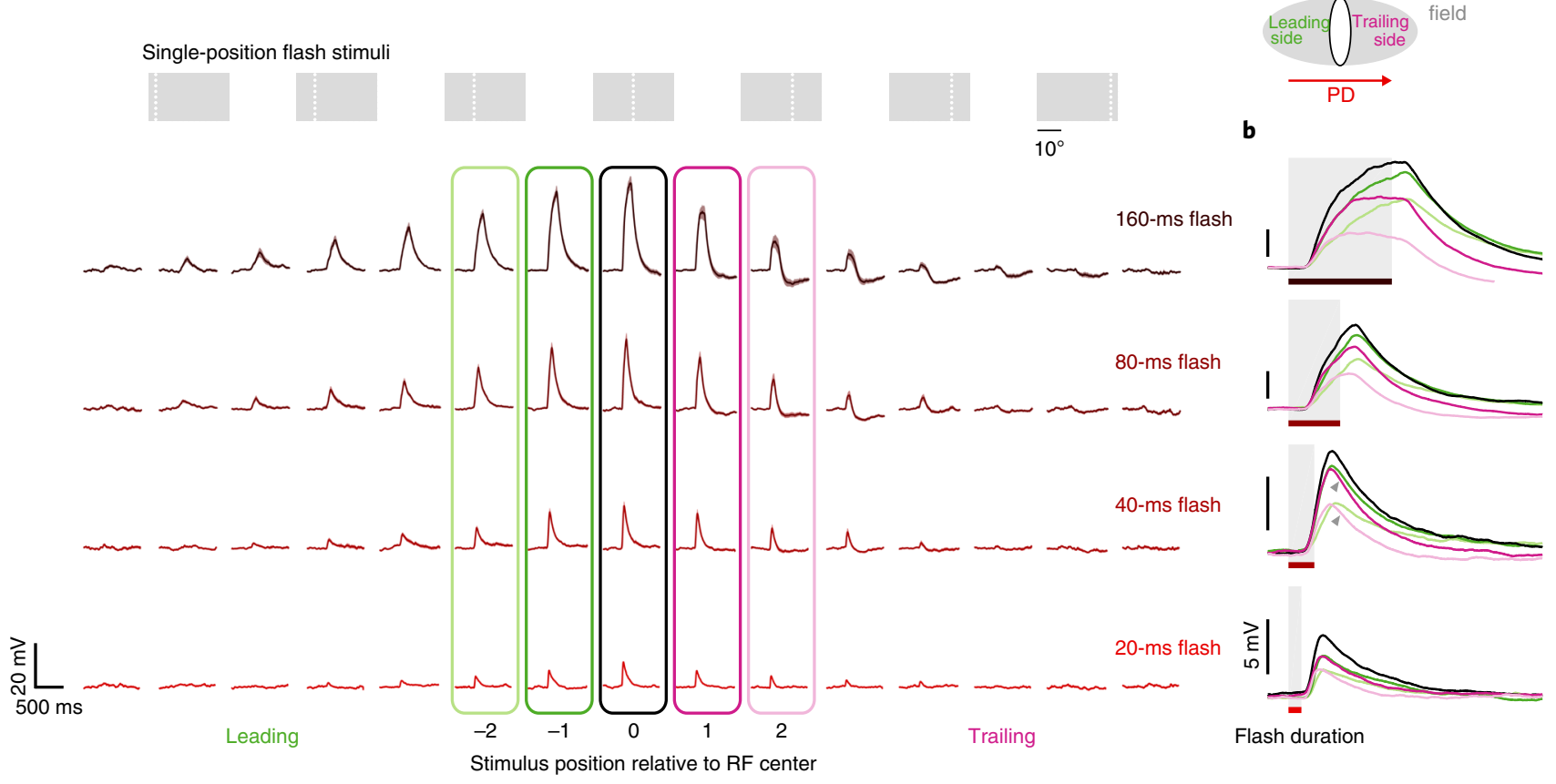

c

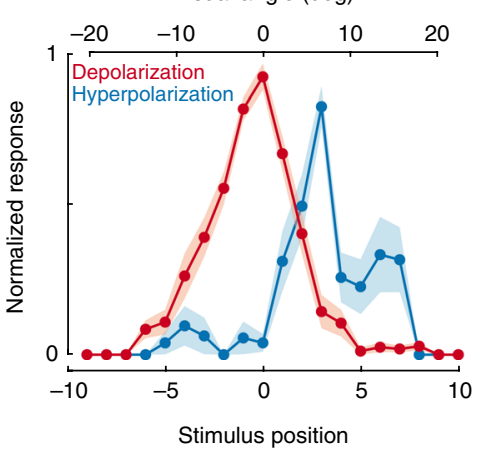

d

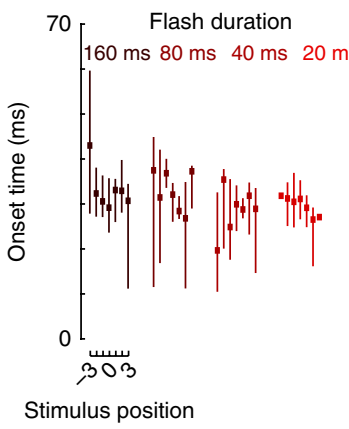

e

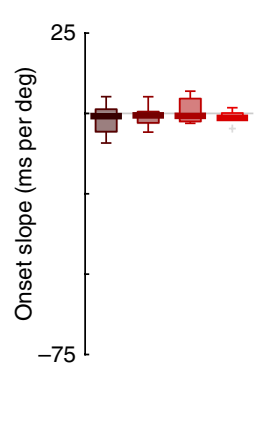

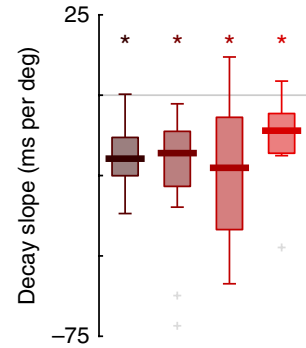

Fig. 2 | T4 cells receive spatially but not temporally offset excitatory and inhibitory inputs. a, Averaged baseline-subtracted responses (mean \pm s.e.m.) to single-position bar flash stimuli (top) along the PD-ND axis of each cell ( $n=17$ cells). $\mathbf{b}$, Mean responses from indicated positions in $\mathbf{a}$, aligned to stimulus presentation (gray rectangles). Arrowheads emphasize differences in decay times. c, Maximum depolarizing and hyperpolarizing responses (mean \pm s.e.m.) by stimulus position for 80 -ms flashes ( $n=17$ cells). $\mathbf{d}$, Response-onset time and decay time to flashes near the RF center (medians and quartiles). Cell numbers vary for each position and duration combination (see Methods). e, Slope of the linear regression of onset time against position and the slope of the decay time against position, calculated separately for each cell and each duration $\left(n=17,17,14,9\right.$ cells; ${ }^{*}$ indicates slope $<0$ with $P<0.01$, one-sided unpaired $t$ test; gray crosses represent outliers; see Methods for boxplot conventions).

The superposition of stationary responses generates directional selectivity. The two-step apparent-motion responses (Fig. 3) suggest that it is the integration of offset excitatory and inhibitory inputs that generates directionally selectivity. However, is this mechanism sufficient to explain the directionally selective responses to moving bars? To address this question, we compared the responses to moving-bar stimuli, comprised of an ordered sequence of single-position bar flashes (Fig. 4a,b), with the superposition of SPFRs (summed after appropriate temporal alignment; Fig. 4c). Notably, the only difference between the summed PD and the summed ND responses was the order in which the SPFRs were aligned. This comparison is conceptually similar to our treatment of the two-step apparent-motion responses but probes more interactions across the entire receptive field. We find that the simple sum of SPFRs (Fig. 4c) captures the essential dynamics of the moving-bar response (Fig. 4c,d) and shows significant directional selectivity at all four speeds (Fig. 4e).
The summed responses approximated the measured responses without any nonlinear integration, an unexpected result as both the $\mathrm{HR}$ and $\mathrm{BL}$ models of motion detection require a nonlinear interaction to compute directional selectivity. To be clear, we are not claiming that directional selectivity emerges from linear operations (for example, the DSI computation is nonlinear); rather we emphasize that these directionally asymmetric responses expose a significant discrepancy between the T4 mechanism and the classical models. To gain further insight, we examined features of the SPFRs for contributions to directional selectivity in the summed responses and found that it is the position-dependent differences (such as the response shape, sign and magnitude) that account for the DSI of the summed responses (Supplementary Fig. 5). However, the summation of aligned SPFRs only accounted for approximately half of the measured T4 DSI (Fig. 4e). Summing negative and positive potentials undercounts the effect of inhibition. For example, in shunting inhibition, a small inhibitory current can eliminate a much larger 
a
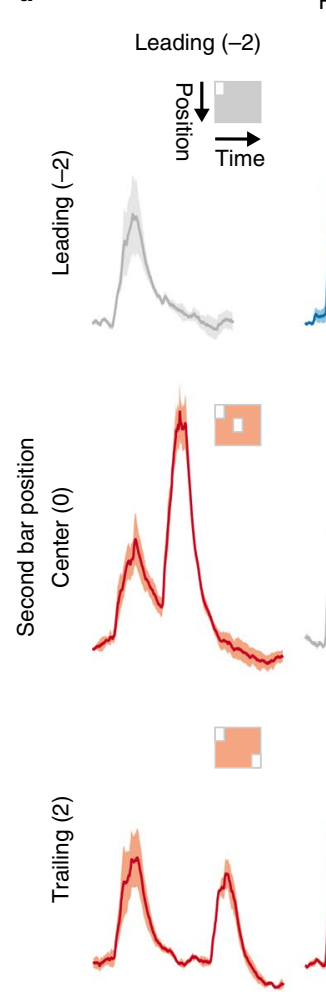

First bar position Center (0)
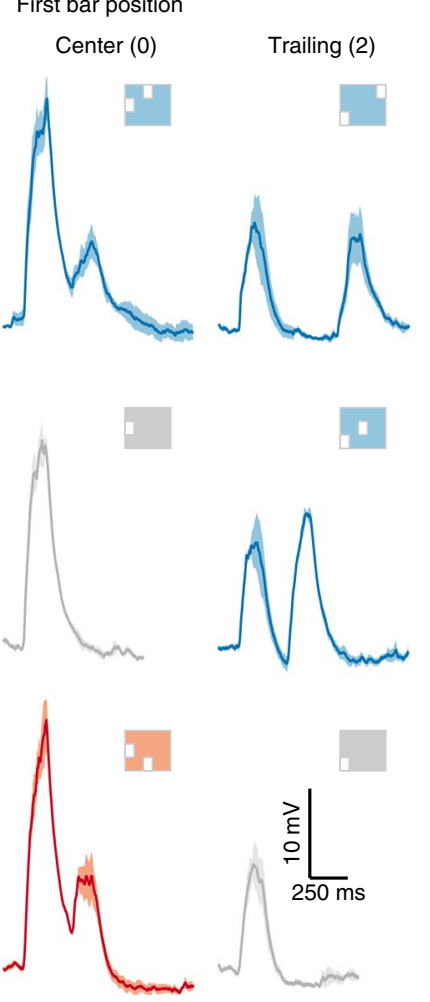

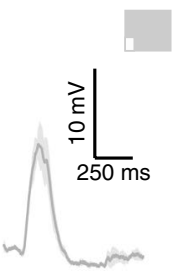

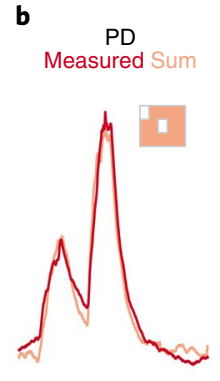
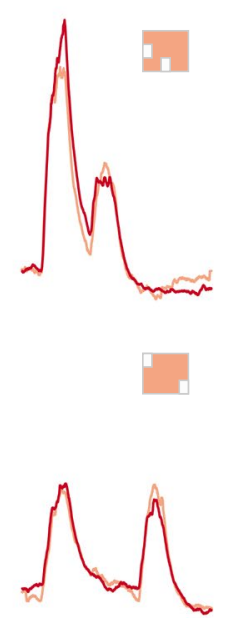

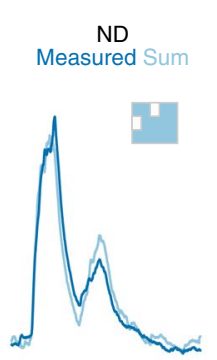
Measured PD ND
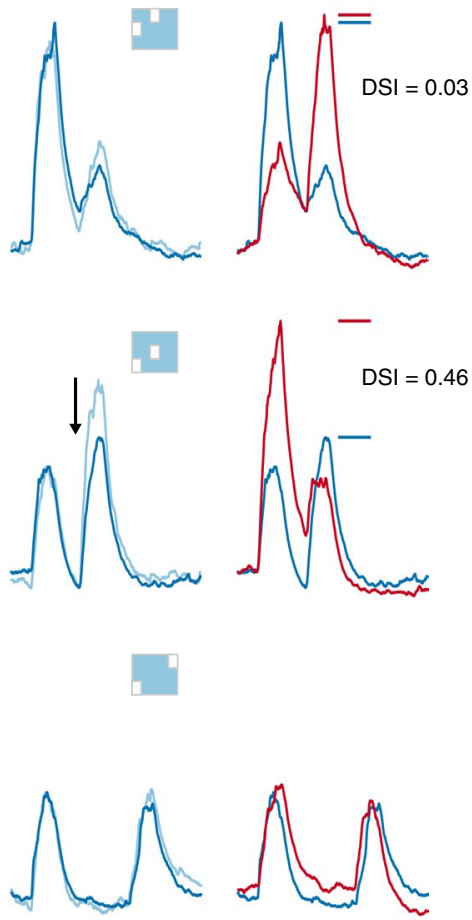

Fig. 3 | T4 responses to two-step apparent motion show ND suppression but not PD enhancement. a, Averaged baseline-subtracted responses (mean \pm s.e.m.) to 160-ms flash stimuli presented along the PD-ND axis ( $n=3$ cells). Single-position responses are shown along the diagonal; PD motion (red) and ND motion (blue) are shown in the lower and upper triangles, respectively. Stimulus positions indicated correspond to those in Fig. 2. Apparent motion is presented as two flash stimuli timed to appear as instances of a bar moving at $14^{\circ} / \mathrm{s}$. Space-time plots of the stimuli are depicted in each inset. b. Comparison of mean measured responses to two-step stimulation to the superposition (time-aligned sum) of the single-position responses. Each row shows responses to stimuli presented at the same positions but in the reversed temporal order. Arrow shows magnitude of ND supression. $\mathbf{c}$, Mean PD and ND responses from the corresponding rows in $\mathbf{b}$ are compared, with DSI indicated. DSI values are averaged from 3 cells (individual values for top panel: 0.02, 0.06, 0.1; for middle panel: 0.47, 0.5, 0.16).

excitatory current. The effect of this undercounting is most prominently seen in the overestimated ND responses (Fig. 4f). Does this mismatch suggest that T4 implements a nonlinear step like the classical models, or could biophysically realistic integration of inhibitory inputs explain the additional measured DSI?

A conductance-based simulation quantitatively predicts the T4 motion response. To answer this question, we built a conductancebased model of a T4 neuron reconstructed from electron microscopy data (Fig. 5a). In our model, we randomly distributed excitatory and inhibitory synapses along the dendritic arbor of the cell but fit the synaptic strengths, the time course of the excitatory and inhibitory conductance changes, and the membrane and axial resistivity using an optimization procedure that minimizes the error between simulated responses to bar flashes and our measured SPFRs (from all positions and all speeds; Fig. 5a and Supplementary Fig. 6a-c). We then used the resulting model to simulate the T4 response to a moving bar (Fig. 5b). Notably, we fit our model's parameters using responses to stationary stimuli (the SPFRs) but used the model to predict the responses to an independent motion stimulus (the moving-bar responses), thus testing the generalization of this model.

In comparison to the summed responses (Fig. 4d), the simulated responses more accurately reproduced the measured responses to both PD and ND motion (Fig. 5b,c). Furthermore, the same simulation qualitatively reproduced the dynamics of T4 responses (Fig. 5b) and quantitatively reproduced the magnitude of the directionally selective responses (Fig. 5c,d and Supplementary Fig. 6d-f) at all tested speeds, suggesting that the same mechanism can account for directional selectivity, even at the fastest speeds tested where hyperpolarization was not directly measured (Fig. 2).

One classical neuronal computation of directional selectivity, based on passive cable properties ${ }^{24}$, is that sequential depolarization directed toward the (thicker) axon generates stronger responses than activation in the opposite direction. To test whether this mechanism could contribute to directional selectivity in T4, we zeroed all inhibitory conductance changes in our model. When the simulation was repeated, only the excitatory synapses were activated as the 'stimulus' swept in the PD or ND. We found that in the absence of inhibition, the DSI of the simulated T4 is abolished at all speeds (Fig. 5d), indicating that depolarizing inputs alone cannot produce directional selectivity. This suggests that inhibitory inputs to T4, in addition to providing the slow hyperpolarization that characterizes responses to slow motion (Fig. 1c), also establish the temporal sharpening of trailing side responses, critical for directional selectivity at faster speeds (Supplementary Fig. 5b).

The striking retinotopic alignment of T4 dendrites with the PD-ND axis was an important anatomical clue in their identification as directionally selective cells ${ }^{9,25}$. We used our simulation to test whether the neuron's morphology substantially contributes to directional selectivity. By positioning all 154 synaptic inputs (inhibitory and excitatory) at the base of the dendrite (all other parameters held constant), we found that these simulation results are almost indistinguishable from the unmodified simulation (Fig. 5d). As a consequence of this result, we expected that a single-compartment 
neuron simulation should also be able to capture the response dynamics of T4. Indeed, this simpler model reproduces the moving-bar responses of $\mathrm{T} 4$ with only negligible differences from the multicompartment simulation results (Fig. 5d). These results suggest that the critical role of the elaborate and directional T4 dendrite is limited to collecting different input signals across a small region of the fly's eye. Taken together, these modeling results corroborate our central finding that inhibitory inputs sculpt the essential asymmetry for directionally selective responses in T4 cells; no additional mechanisms are required.

\section{Discussion}

We used intracellular recordings to probe the mechanism of directionally selectivity for $\mathrm{ON}$ motion in T4 neurons. Using online stimulus generation, we finely mapped the receptive field of $\mathrm{T} 4$ neurons (Fig. 2). Notably, this RF structure, comprised of responses to stationary ON bars, can be used to predict the response of T4 to moving bars (Fig. 4). We then improved these predictions with a parsimonious biophysical model integrating offset excitatory and inhibitory conductances (Fig. 5).
In the present study, we show that directional selectivity in T4 neurons arises solely from ND suppression. Recent studies that used calcium imaging of T4 neuron responses to a two-step apparentmotion stimulus found evidence for PD enhancement ${ }^{18,19}$ or for both PD enhancement and ND suppression ${ }^{20,22}$. These discrepancies may arise from differences in the stimulus design, differences between single-neuron and population recordings, differences between measurements of membrane potential versus calcium indicator fluorescence, or from combinations of these factors. Since the transformation between membrane potential and calcium indicator fluorescence is likely to be superlinear ${ }^{21}$, measured calcium responses may appear enhanced when compared to their summed components, even when the underlying voltage response does not show this enhancement (Supplementary Fig. 7). However, as Fisher et al. ${ }^{18}$ controlled for this possibility, we believe that the difference is most likely explained by differences in stimulus design (centered bars versus edges ${ }^{18}$; centered narrow bars versus centered wider spots ${ }^{20}$ ). Haag et al. ${ }^{20}$ found PD enhancement in T4 cells, but only for stationary stimuli wider than those used here. These larger stimuli, in addition to strongly depolarizing the measured T4 cells, a

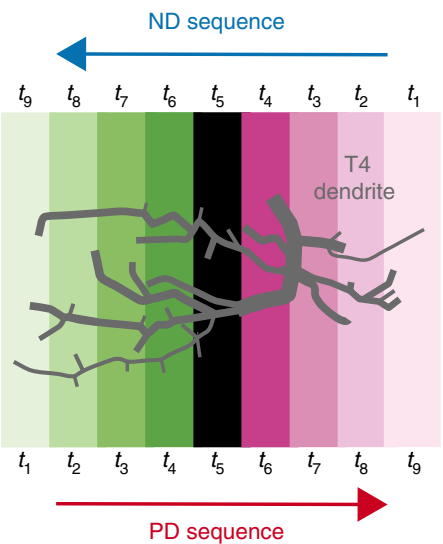

b Measured moving bar $(28 \%$ s) response (cell 15)

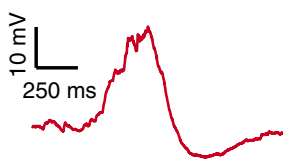

d

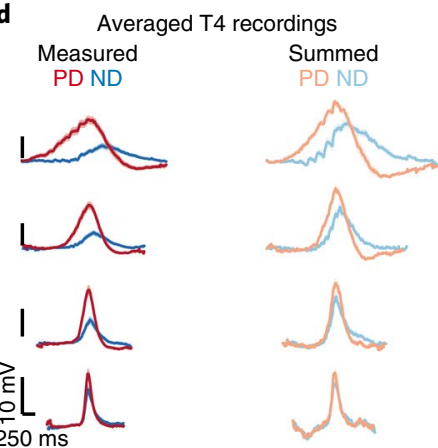

C
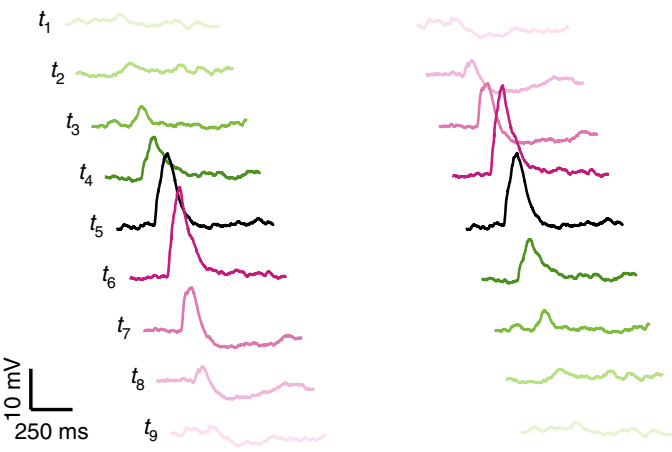

Summed SPFRs
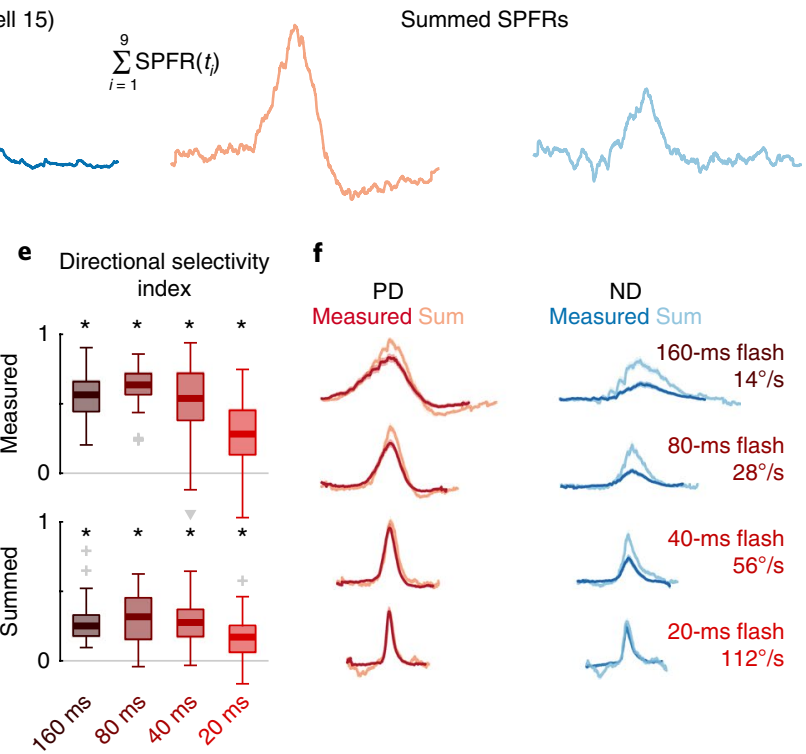

ND
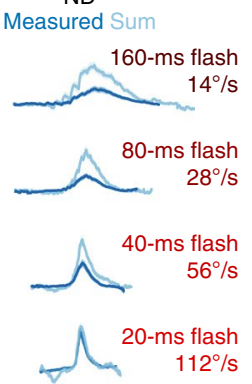

Fig. 4 | Ordered summation of SPFR qualitatively reproduces moving bar responses. a, Schematized T4 dendrites overlaid on nine bar-stimulus positions (colors as in Fig. 2). b, Mean PD and ND motion responses from an example cell to the $28^{\circ} / \mathrm{s}$ bar motion. c, Top: SPFRs from the example cell in $\mathbf{b}$ colored by position, temporally aligned to account for moving-bar position. Bottom: summed SPFRs for PD and ND motion. $\mathbf{d}$, Comparison of mean measured moving bar responses and mean summed SPFRs ( $n=31$ trajectories, 16 cells). e, Boxplots of DSI values for measured and summed responses across speeds (mean \pm s.e.m.; $n=31$ trials, 16 cells; see Methods for boxplot conventions). Crosses represent outliers; triangle denotes a point outside the scope of the plot ( ${ }^{*}$ indicates DSI $>0$ with $P<0.01$, one-sided unpaired $t$ test). f, As in $\mathbf{d}$ but juxtaposing measured responses and summed SPFRs for PD and ND motion separately (mean \pm s.e.m.). 
also effectively stimulate neighboring T4 cells. But since T4 neurons synapse onto other T4 cells with exquisite precision-only cells with the same directional tuning are connected and only along the direction of preferred motion ${ }^{17}$ - this circuit mechanism should produce a superlinear response in one direction (along the PD), but not in the opposite direction. This enhancement of PD motion over a slightly larger spatial scale appears to be evidence for directed facilitation from neighboring T4 cells but not for an HR-like mechanism responsible for directional selectivity (since blocking synaptic transmission in T4 neurons does not substantially reduce directionally selective responses in T4 cells ${ }^{15,22}$ ). This factor may partially explain the measurement of PD enhancement in Fisher et al. ${ }^{18}$, but future experiments will be required to clarify these discrepancies.

Anatomical studies have shown that dendrites of T4 cells are aligned with the $\mathrm{PD}^{9,25}$ and receive spatially offset synaptic inputs from distinct columnar neuron types ${ }^{17}$. The arbors span several (3-4) retinotopic columns in the medulla ${ }^{16}$, which, given the $\sim 5^{\circ}$ visual angle per column, corresponds well to our measured RF width of $\sim 20^{\circ}$ (Fig. 2). The cholinergic columnar neurons Mil and $\mathrm{Tm} 3$ preferentially synapse onto T4 in the central to distal part of the arbor, while the GABAergic neurons Mi4, C3 and CT1 synapse mainly onto the base of the dendrite ${ }^{15,17,26,27}$. This anatomical arrangement agrees remarkably well with the functional organization of our measured $\mathrm{T} 4$ receptive field, with leading-side excitation and trailing-side inhibition.

A recent study from our lab used circuit perturbations to also propose a hybrid mechanism for T4 motion responses ${ }^{15}$. Although we showed nonlinear integration of excitatory inputs by $\mathrm{T} 4$, we did not show that this nonlinearity contributes to the computation of directional selectivity, so these results do not conflict with the current findings. In fact, electron microscopy-based circuit analysis ${ }^{9,17}$, as well as a number of functional studies ${ }^{14,15,23,26,28,29}$, suggests that multiple cell types (at least Mil and Tm3) contribute to the excitatory $\mathrm{ON}$ component of our measured RF, without evidence for a spatial offset required for an HR-like mechanism ${ }^{17}$. In our previous study, we also showed that silencing one of the main inhibitory inputs to T4 (Mi4) does not reduce directional selectivity ${ }^{15}$. Since our current results strongly suggest that inhibition is necessary for computing directional selectivity, it is entirely possible that the critical inhibitory inputs to T4 are not simply contributed by a single cell type (other candidates include CT1, C3 and TmY15 ${ }^{17}$ ).

Synthesis of experimental results with classical computational models for motion detection. Historically, the computation of directional selectivity has been conceptualized with algorithmic models outlining the general properties that transform nonselective inputs into a directionally selective output. Here we compare our results with the implications of the classical models and present an algorithmic summary of our findings. Motion-detector models typically describe a 'fully opponent' computation, whereby local motion estimates in opposing directions are subtracted to minimize nonmotion signals ${ }^{1}$. Since T4 is now understood to reside one step before this opponent subtraction ${ }^{30}$, we only consider the subunits of each model type before this subtractions stage. The classical model for insect motion detection is the HR detector ${ }^{2}$. This detector generates directional selectivity by delaying leading-side excitatory input and combining it with trailing-side excitatory input with a nonlinear amplification, thus enhancing the PD response (Fig. 6a). If T4 directional selectivity was based on an HR-like mechanism, we would expect to find (i) excitatory inputs spanning the receptive field, (ii) differing temporal filtering of inputs resulting in a delayed leading side and (iii) enhanced responses to PD motion (Fig. 6a). We did not find evidence for any of these properties: (i) T4 receives offset excitatory and inhibitory inputs (Fig. 2c), (ii) leading side responses are not temporally delayed (Fig. 2d, e and Supplementary Fig. 8), and (iii) PD motion is not enhanced (Figs. 3, 4 and 5).

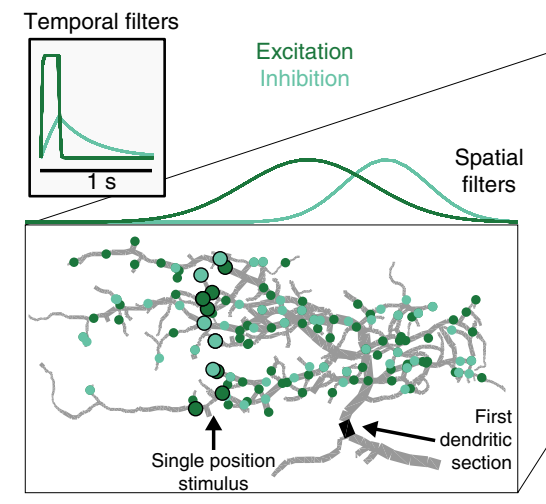

b
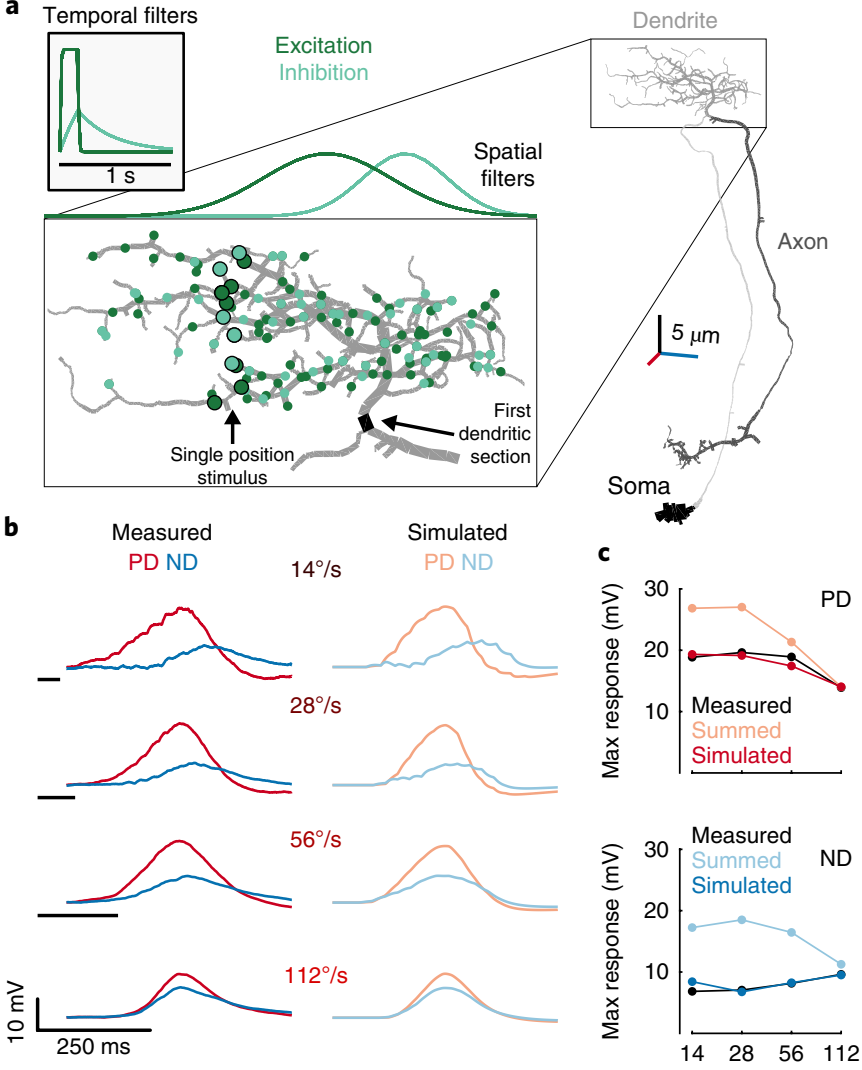

Stimulus speed $(\%)$
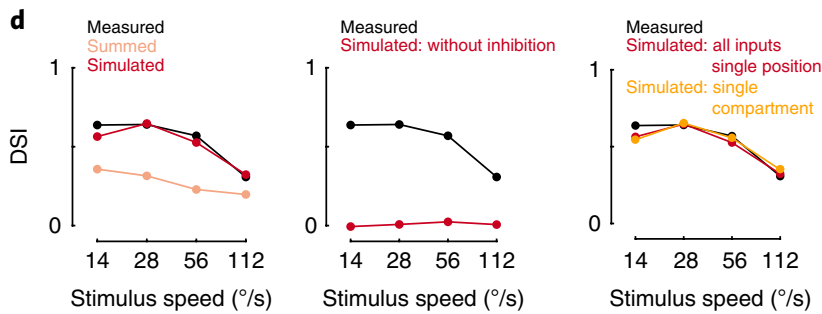

Fig. 5 | Asymmetric inputs in a conductance-based model predict directionally selective responses. a, Anatomical reconstruction of a T4 cell used in the simulation, with expanded dendrite showing positions of modeled excitatory (dark green) and inhibitory (light green) synapses. Enlarged markers indicate active synapses for an example stimulus position. Spatial filters, which determine synaptic weight, are shown normalized and aligned to the dendrite, and temporal filters are shown in inset. $\mathbf{b}$, Mean measured motion responses (from Fig. 4) compared to the simulated predictions. c, Peak PD and ND responses measured in T4s, compared to the summed SPFRs (from Fig. 4) and simulation results. d, DSI for the mean measured responses compared to: (left) model simulation results and summed responses; (center) simulation results without inhibition; (right) simulation results with all synaptic inputs placed in same location, and a separate single-compartment simulation (detailed in Methods).

Furthermore, the excitatory region of the receptive field is motion blind (Supplementary Fig. 4).

An alternative classical model is the BL detector ${ }^{3}$, which integrates fast leading-side excitation and slower trailing-side inhibition through the nonlinear 'AND-NOT' operation (Fig. 6b). Directional selectivity is computed when excitatory input precedes the inhibitory input; in the reverse order, ND motion is suppressed. We find several properties in our T4 recordings that agree with this framework: (i) leading-side excitatory responses and trailing-side inhibitory responses (Fig. 2), (ii) longer temporal filtering on the trailing side, such that 


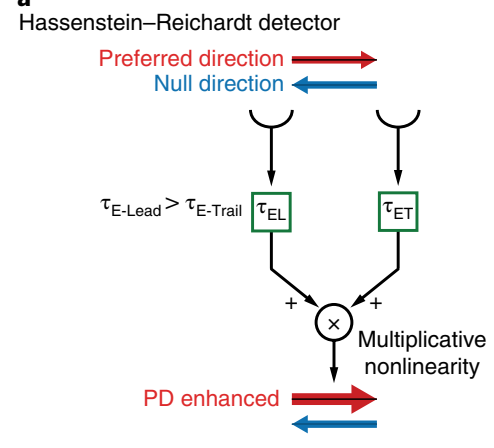

barlow-Levick detector

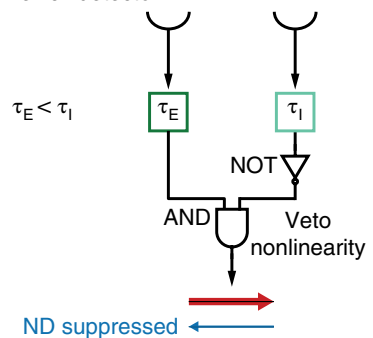

Adelson-Bergen detector

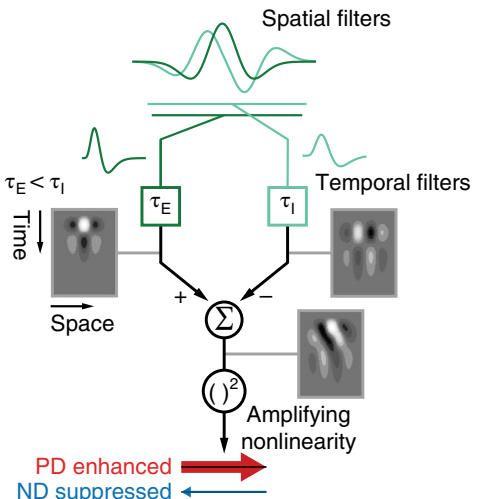

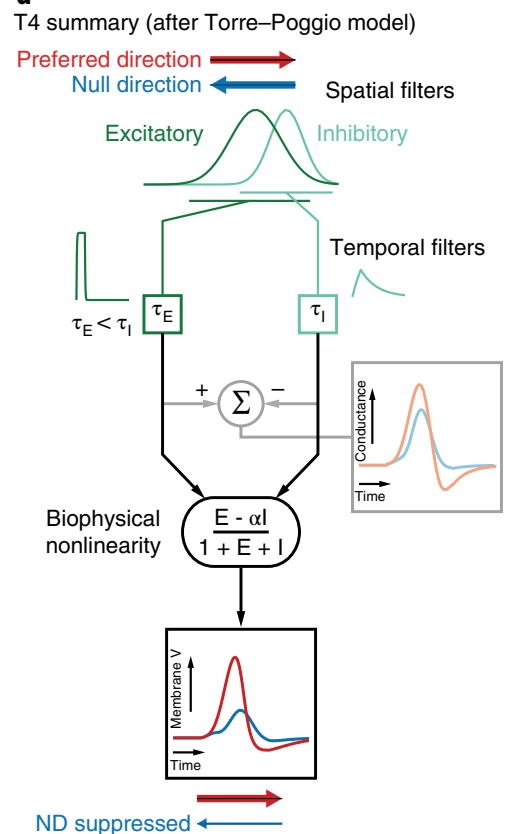

Fig. 6 | Comparison of the T4 mechanism with classical computational models for directional selectivity. $\mathbf{a}$, The HR detector ${ }^{2}$ uses slower filtering on the leading side arm and a multiplicative nonlinearity to produce coincidence detection of excitatory signals. The time constants are labeled as $\boldsymbol{\tau}_{\mathrm{E}-\text { Lead }}$ and $\boldsymbol{\tau}_{\mathrm{E}-\text { Trail }}$, where $\mathrm{E}$ indicates filtering of an excitatory leading (lead, $\mathrm{L}$ ) or trailing (trail, $\mathrm{T}$ ) signal. The semicircles represent two neighboring inputs to the motion detectors. b. The BL detector ${ }^{3}$ combines a slow trailing-side inhibitory (I) arm with a faster leading-side excitatory (E) arm through an AND-NOT operation that vetoes motion in the null direction. $\mathbf{c}$, The $A B$ detector ${ }^{31}$ uses oriented spatiotemporal filters, created from offset spatial and fast and slow temporal filters, to produce directional selectivity. $\mathbf{d}$, A summary of the T4 mechanism formatted for comparison to the algorithmic models, based on the TorrePoggio model ${ }^{35}$. The simple RF structure of T4 (Supplementary Fig. 8) does not require the more complex spatial and temporal filters used in the AB model. Instead we use filters fit to T4 measurements (Fig. 5), which produce a fast, excitatory leading signal and a delayed, inhibitory trailing signal. As in the AB model, directionally selective responses can be obtained through a linear combination of these signals (gray lines). The dynamic nonlinearity operates on the magnitude of excitatory (E) and inhibitory (I) conductances (detailed in Methods) to produce a directionally selective membrane potential response.

inhibition outlasts excitation (Figs. 2 and 5 and Supplementary Fig. 8) and (iii) clear evidence for ND suppression with both two-step and moving-bar apparent motion (Figs. 3c and 4f).

While the organization of the T4 RF agrees with the algorithmic principle of the BL model, we find an important difference in the integration mechanism. The BL model uses a strong nonlinearity and so does not produce directionally selective responses from the superposition of the responses to stationary inputs (which our T4 recordings show; Figs. 3 and 4). This seemingly technical distinction is in fact quite consequential, because in both the HR and BL models, the nonlinearity is a defining characteristic: without it, there are no directionally selective responses. In contrast, our T4 results show that directional selectivity is already present in the superposition of the stationary stimulus responses.

A third class of motion detectors-the Adelson-Bergen (AB) motion-energy model ${ }^{31}$ - generates directional selectivity using filters that are oriented in space and time. These linear filters are combined spatial and temporal filters that feature a prominent space-time tilt indicating the preferred direction. In Fig. $6 \mathrm{c}$ we present only the relevant subunit of the full $A B$ model for comparison with our data. This subunit has (i) different spatial filters on each of its arms, (ii) excitatory and inhibitory inputs, (iii) a linear integration step that produces a directionally selective response from the superposition of stationary responses (as seen in simple cells of the cat visual cortex ${ }^{32}$ ) and (iv) a static, amplifying nonlinearity. This flexible model, whose commonalities with variants of the classical motion-detection models have been broadly discussed ${ }^{1,31,33}$, provides a useful framework for representing the $\mathrm{T} 4$ computation.
In light of the commonalities and discrepancies between classical algorithmic models and our results, we next provide a summary of our understanding of T4 in a format that is directly comparable to these models (Fig. 6d), with an excitatory and an inhibitory arm. The more complex spatial and temporal filters of the $A B$ model, selected to match cortical recordings, are replaced with the filters describing T4 responses (Fig. 5 and Methods). The spatial filters create the leading-versus-trailing offset and the temporal filters create the fastversus-slow difference between the excitatory and inhibitory arms. In contrast to the 'point-sampling' inputs of the classical HR and BL models, T4 shows spatially extended responses to visual input (Fig. 2b), which has previously been shown to improve the accuracy of motion detection ${ }^{34}$. While the sum of the linear filter outputs produces asymmetric responses to PD and ND motion (Fig. 6d), T4 neurons further enhance this selectivity through nonlinear integration. While the $\mathrm{AB}$ model employs a static, amplifying nonlinearity, we measure a suppressing nonlinearity that is well approximated with a simplified passive, biophysical model (similar to a previous formulation $^{35}$; Figs. 5d and 6d, and see Methods).

Spatiotemporal receptive fields of T4 cells. Representing the response properties of neurons using spatiotemporal receptive fields, equivalent to the responses of the spatiotemporal filters in the $\mathrm{AB}$ model, has provided significant insight into transformation along the visual pathway of mammals ${ }^{36}$ and has recently also been applied to the responses of directionally selective neurons in flies ${ }^{19,21}$. We replotted the averaged SPFRs to approximate the spatiotemporal receptive field of a T4 neuron (Supplementary Fig. 8). As expected for a directionally selective neuron, we found a distinctive tilt in this receptive field, 
indicating the PD. This RF has both excitatory and inhibitory lobes, which both appear tilted, an organization that may suggest a PD-enhancing and ND-suppressing mechanism ${ }^{19,21}$. To explore the basis of the RF tilt, we used our multicompartment T4 model and replotted the simulated SPFRs (Supplementary Fig. 6c) as a spatiotemporal RF (Supplementary Fig. 8). Since the model was fit to the SPFR data, the qualitative agreement between these RFs is not surprising. The simulated RF also features a tilted excitatory lobe. When we removed inhibition in our model (Fig. 5d) and reproduced the RF (Supplementary Fig. 8), we found that the excitatory lobe was no longer tilted, consistent with our inference that inhibition sharpens trailingside responses (Fig. 2b). This simulation shows that a tilted, two-lobed spatiotemporal RF does not necessarily support a hybrid mechanism and reiterates the lack of evidence in our data for PD enhancement.

It is worth noting that the receptive fields of T5 cells, the OFF directionally selective neurons, look qualitatively similar to those estimated for T4 neurons ${ }^{19-21}$. Therefore, it is possible that T5 neurons compute directional selectivity using the same simple mechanism that we have established here for T4 neurons. Notably, no small-field inhibitory inputs $\mathrm{s}^{37,38}$ to $\mathrm{T} 5$ have thus far been identified, raising the possibility of different implementations of the same motion computation in ON and OFF pathways ${ }^{39,40}$.

\section{Methods}

Methods, including statements of data availability and any associated accession codes and references, are available at https://doi. org/10.1038/s41593-017-0046-4.

Received: 11 April 2017; Accepted: 6 November 2017; Published online: 8 January 2018

\section{References}

1. Borst, A. \& Egelhaaf, M. Principles of visual motion detection. Trends Neurosci. 12, 297-306 (1989)

2. Hassenstein, V. B. \& Reichardt, W. Systemtheoretische Analyse der Zeit-, Reihenfolgen- und Vorzeichenauswertung bei der Bewegungsperzeption des Rüsselkäfers Chlorophanus. Z. Naturforsch. B 11, 513-524 (1956).

3. Barlow, H. B. \& Levick, W. R. The mechanism of directionally selective units in rabbit's retina. J. Physiol. (Lond.) 178, 477-504 (1965).

4. Poggio, T. \& Reichardt, W. Considerations on models of movement detection. Kybernetik 13, 223-227 (1973).

5. Buchner, E. Elementary movement detectors in an insect visual system. Biol. Cybern. 24, 85-101 (1976).

6. Tuthill, J. C., Chiappe, M. E. \& Reiser, M. B. Neural correlates of illusory motion perception in Drosophila. Proc. Natl. Acad. Sci. USA 108, 9685-9690 (2011).

7. Haag, J., Denk, W. \& Borst, A. Fly motion vision is based on Reichardt detectors regardless of the signal-to-noise ratio. Proc. Natl. Acad. Sci. USA 101, 16333-16338 (2004).

8. Borst, A., Flanagin, V. L. \& Sompolinsky, H. Adaptation without parameter change: dynamic gain control in motion detection. Proc. Natl. Acad. Sci. USA $102,6172-6176(2005)$

9. Takemura, S. Y. et al. A visual motion detection circuit suggested by Drosophila connectomics. Nature 500, 175-181 (2013).

10. Strother, J. A., Nern, A. \& Reiser, M. B. Direct observation of ON and OFF pathways in the Drosophila visual system. Curr. Biol. 24, 976-983 (2014).

11. Maisak, M. S. et al. A directional tuning map of Drosophila elementary motion detectors. Nature 500, 212-216 (2013).

12. Joesch, M., Schnell, B., Raghu, S. V., Reiff, D. F. \& Borst, A. ON and OFF pathways in Drosophila motion vision. Nature 468, 300-304 (2010).

13. Clark, D. A., Bursztyn, L., Horowitz, M. A., Schnitzer, M. J. \& Clandinin, T. R. Defining the computational structure of the motion detector in Drosophila. Neuron 70, 1165-1177 (2011).

14. Behnia, R., Clark, D. A., Carter, A. G., Clandinin, T. R. \& Desplan, C. Processing properties of $\mathrm{ON}$ and OFF pathways for Drosophila motion detection. Nature 512, 427-430 (2014).

15. Strother, J. A. et al. The emergence of directional selectivity in the visual motion pathway of Drosophila. Neuron 94, 168-182.e10 (2017).

16. Fischbach, K.-F. \& Dittrich, A. The optic lobe of Drosophila melanogaster. I. A Golgi analysis of wild-type structure. Cell Tissue Res. 258, 441-475 (1989).

17. Takemura, S. Y. et al. The comprehensive connectome of a neural substrate for 'ON' motion detection in Drosophila. eLife https://doi.org/10.7554/ eLife.24394 (2017).

18. Fisher, Y. E., Silies, M. \& Clandinin, T. R. Orientation selectivity sharpens motion detection in Drosophila. Neuron 88, 390-402 (2015).
19. Salazar-Gatzimas, E. et al. Direct measurement of correlation responses in Drosophila elementary motion detectors reveals fast timescale tuning. Neuron 92, 227-239 (2016).

20. Haag, J., Mishra, A. \& Borst, A. A common directional tuning mechanism of Drosophila motion-sensing neurons in the ON and in the OFF pathway. elife 6, e29044 (2017).

21. Leong, J. C., Esch, J. J., Poole, B., Ganguli, S. \& Clandinin, T. R. Direction selectivity in Drosophila emerges from preferred-direction enhancement and null-direction suppression. J. Neurosci. 36, 8078-8092 (2016).

22. Haag, J., Arenz, A., Serbe, E., Gabbiani, F. \& Borst, A. Complementary mechanisms create direction selectivity in the fly. eLife 5, e17421 (2016).

23. Arenz, A., Drews, M. S., Richter, F. G., Ammer, G. \& Borst, A. The temporal tuning of the Drosophila motion detectors is determined by the dynamics of their input elements. Curr. Biol. 27, 929-944 (2017).

24. Rall, W. Theoretical significance of dendritic trees for neuronal input-output relations. in Neural Theory and Modeling 7397 (ed. Reiss, R. F.) (Stanford University Press, Palo Alto, 1964).

25. Strausfeld, N. J. \& Lee, J. K. Neuronal basis for parallel visual processing in the fly. Vis. Neurosci. 7, 13-33 (1991).

26. Pankova, K. \& Borst, A. Transgenic line for the identification of cholinergic release sites in Drosophila melanogaster. J. Exp. Biol. 220, 1405-1410 (2017).

27. Long, X., Colonell, J., Wong, A. M., Singer, R. H. \& Lionnet, T. Quantitative mRNA imaging throughout the entire Drosophila brain. Nat. Methods 14, 703-706 10 (2017).

28. Yang, H. H. et al. Subcellular imaging of voltage and calcium signals reveals neural processing in vivo. Cell 166, 245-257 (2016).

29. Ammer, G., Leonhardt, A., Bahl, A., Dickson, B. J. \& Borst, A. Functional specialization of neural input elements to the Drosophila ON motion detector. Curr. Biol. 25, 2247-2253 (2015).

30. Mauss, A. S. et al. Neural circuit to integrate opposing motions in the visual field. Cell 162, 351-362 (2015).

31. Adelson, E. H. \& Bergen, J. R. Spatiotemporal energy models for the perception of motion. J. Opt. Soc. Am. A 2, 284-299 (1985).

32. Jagadeesh, B., Wheat, H. S. \& Ferster, D. Linearity of summation of synaptic potentials underlying direction selectivity in simple cells of the cat visual cortex. Science 262, 1901-1904 (1993).

33. van Santen, J. P. \& Sperling, G. Elaborated Reichardt detectors. J. Opt. Soc. Am. A 2, 300-321 (1985).

34. Dror, R. O., O'Carroll, D. C. \& Laughlin, S. B. Accuracy of velocity estimation by Reichardt correlators. J. Opt. Soc. Am. A Opt. Image Sci. Vis 18, 241-252 (2001).

35. Torre, V. \& Poggio, T. Synaptic mechanism possibly underlying directional selectivity to motion. Proc. R. Soc. Ser. B Biol. Sci. https://doi.org/10.1098/ rspb.1978.0075 (1978).

36. DeAngelis, G. C., Ohzawa, I. \& Freeman, R. D. Receptive-field dynamics in the central visual pathways. Trends Neurosci. 18, 451-458 (1995).

37. Serbe, E., Meier, M., Leonhardt, A. \& Borst, A. Comprehensive characterization of the major presynaptic elements to the Drosophila OFF motion detector. Neuron 89, 829-841 (2016).

38. Shinomiya, K. et al. Candidate neural substrates for off-edge motion detection in Drosophila. Curr. Biol 24, 1062-1070 (2014).

39. Fitzgerald, J. E. \& Clark, D. A. Nonlinear circuits for naturalistic visual motion estimation. eLife 4, e09123 (2015).

40. Leonhardt, A. et al. Asymmetry of Drosophila ON and OFF motion detectors enhances real-world velocity estimation. Nat. Neurosci. 19, 706-715 (2016).

\section{Acknowledgements}

We thank A. Nern for providing the driver line and image, K. Shinomiya and Janelia's FlyEM project team for providing the reconstructed T4 cell morphology, M. Cembrowski for assistance with the NEURON simulations and E. Rogers for help with fly husbandry. We are also grateful to A. Hermundstad, J. Dudman, N. Spruston, B. Mensh and members of the Reiser lab for comments on the manuscript. This project was supported by the Howard Hughes Medical Institute.

\section{Author contributions}

E.G. and M.B.R. designed experiments; E.G. performed experiments and analysis. E.G. and S.R. conducted the simulation study. E.G. and M.B.R. wrote the manuscript.

\section{Competing interests}

The authors declare no competing financial interests.

\section{Additional information}

Supplementary information is available for this paper at https://doi.org/10.1038/ s41593-017-0046-4.

Reprints and permissions information is available at www.nature.com/reprints. Correspondence and requests for materials should be addressed to M.B.R.

Publisher's note: Springer Nature remains neutral with regard to jurisdictional claims in published maps and institutional affiliations. 


\section{Methods}

Histology. To visualize the expression pattern of the T4 driver line (SS02344), brains of female flies were immunolabeled and imaged as described ${ }^{41}$. Anti-Brp was used as a stain for the neuropil marker (anti-nc82, 1:30, Developmental Studies Hybridoma Bank) and pJFRC225-5XUAS-IVS-myr::smFLAG (rat antiFLAG, 1:100, Novus Biologicals) in VK00005 ${ }^{42}$ was used as the reporter for GAL4 expression. The image shown in Supplementary Fig. la was generated from a confocal stack imaged on a Zeiss LSM 710 microscope with a $63 \times$ objective, and resampled using Vaa3D $\mathrm{D}^{43}$

\section{Electrophysiology. Experiments were performed on 1- to 2-d old female} Drosophila melanogaster (flies were reared under constant light conditions at $24^{\circ} \mathrm{C}$ some flies experienced periods of darkness, including overnight, before dissection) To target T4 cells, a single genotype was used: pJFRC28-10XUAS-IVS-GFP-p10 $0^{44}$ in attP2 crossed to stable split-GAL4 SS02344 (VT015785-p65ADZp (attP40); R42F06-ZpGdbd (attP2), generously provided by A. Nern in G. Rubin's laboratory, Janelia; line details with expression data available from http://splitgal4.janelia.org/). Flies were briefly anesthetized on ice and transferred to a special chilled vacuum holder where they were mounted, with the head tilted down, to a customized platform machined from PEEK using UV-cured glue (Loctite 3972). CAD files for the platform and vacuum holder are available upon request. To reduce brain motion, the proboscis was fixed to the head with a small amount of the same glue. The posterior part of the cuticle was removed using syringe needles and fine forceps. The perineural sheath was peeled using fine forceps and, if needed, further removed with a suction pipette under the microscope. To further reduce brain motion, muscle $16^{45}$ was removed from between the antenna.

The brain was continuously perfused with an extracellular saline containing (in mM): $103 \mathrm{NaCl}, 3 \mathrm{KCl}, 1.5 \mathrm{CaCl}_{2}, 4 \mathrm{MgCl}_{2}, 1 \mathrm{NaH}_{2} \mathrm{PO}_{4}, 26 \mathrm{NaHCO}_{3}, 5$ $\mathrm{N}$-Tris (hydroxymethyl) methyl-2-aminoethane-sulfonic acid, 10 glucose and 10 trehalose $\mathrm{f}^{46}$. Osmolarity was adjusted to $275 \mathrm{mOsm}$, and saline was bubbled with $95 \% \mathrm{O}_{2} / 5 \% \mathrm{CO}_{2}$ during the experiment to reach a final $\mathrm{pH}$ of 7.3. Pressurepolished patch-clamp electrodes were pulled for a resistance of 9.5-10.5 M $\Omega$ and filled with an intracellular saline containing (in mM): 140 KAsp, 10 HEPES, 1.1 EGTA, $0.1 \mathrm{CaCl}_{2}$, $4 \mathrm{MgATP}, 0.5 \mathrm{NaGTP}$ and 5 glutathione ${ }^{46}$. We added $250 \mu \mathrm{M}$ Alexa Fluor 594 hydrazide to the intracellular saline before each experiment, to reach a final osmolarity of $265 \mathrm{mOsm}$, with a $\mathrm{pH}$ of 7.3 .

The mounted, dissected flies were positioned on a rigid platform mounted on an air table. Recordings were obtained from labeled $\mathrm{T} 4$ cell bodies under visual control using a Sutter SOM microscope with a $60 \times$ water-immersion objective. To visualize the GFP-labeled cells, a monochrome, IR-sensitive CCD camera (ThorLabs 1500M-GE) was mounted to the microscope, an IR LED provided oblique illumination (ThorLabs M850F2), and a 460-nm LED provided GFP excitation (Sutter TLED source). Images were acquired using Micro-Manager ${ }^{47}$ to allow for automatic contrast adjustment.

All recordings were obtained from the left side of the brain. Current-clamp recordings were low-pass filtered at $10 \mathrm{kHz}$ using an Axon MultiClamp 700B amplifier, and were sampled at $20 \mathrm{kHz}$ (National Instrument PCIe-7842R LX50 Multifunction RIO board) using custom LabView (2013 v.13.0.1f2; National Instruments) and Matlab (2015a; MathWorks) software. The membrane potential of recorded cells was set around $-65 \mathrm{mV}$ (uncorrected for liquid junction potential), which required injecting a small, hyperpolarizing current ( $0-3 \mathrm{pA})$, which after initial adjustment was maintained at a constant value throughout the recording. To verify recording quality, current-step injections were performed intermittently throughout the experiment. Recordings from cells in which either visual or current-step responses diminished noticeably were terminated.

Visual stimuli. The display was constructed from an updated version of the LED panels previously described ${ }^{48}$. The arena covered slightly more than one half of a cylinder ( $216^{\circ}$ in azimuth and $\sim 72^{\circ}$ in elevation) of the fly's visual field, with the maximum pixel diameter subtending an angle of $\sim 2.25^{\circ}$ on the fly eye. Green LEDs (emission peak: $565 \mathrm{~nm}$ ) were used; bright stimuli were $~ 72 \mathrm{~cd} / \mathrm{m}^{2}$ and were presented on an intermediate intensity background of $\sim 31 \mathrm{~cd} / \mathrm{m}^{2}$.

Visual stimuli were generated using custom-written Matlab code that allowed rapid generation of stimuli based on individual cell responses. In contrast to the published stimulus control system ${ }^{48}$, we have now implemented an FPGA-based panel display controller, using the same PCIe card (National Instrument PCIe7842R LX50 Multifunction RIO board) that also acquired the electrophysiology data. This new control system (implemented in LabView) streams pattern data directly from PC file storage, allowing for online stimulus generation. Furthermore, this new control system featured high-precision (to $10 \mu \mathrm{s}$ ) timing and logging of all events, enabling reliable alignment of electrophysiology data with visual stimuli.

To map the receptive field (RF) center of each recorded cell, three grids of flashing bright squares (on an intermediate intensity background) were presented at increasing resolution. Each flash stimulus was presented for $140 \mathrm{~ms}$. First, a $6 \times 7$ grid of non-overlapping bright squares $\left(5 \times 5 \mathrm{LEDs} ; \sim 11^{\circ} \times \sim 11^{\circ}\right)$ was presented. If a response was detected, a denser $3 \times 3$ grid with $50 \%$ overlapping bright and dark squares $\left(5 \times 5 \mathrm{LEDs} ; \sim 11^{\circ} \times \sim 11^{\circ}\right)$, to further verify that these were T4 cells, was presented at the estimated position of the RF center. The dark squares were used to further verify that the recorded cells were T4, and not T5, cells (see Supplementary
Fig. 1). If a recorded cell was consistently responsive to the first two mapping stimuli, a third one was presented to identify the RF center. A $5 \times 5$ grid of $3 \times 3$ LED bright squares separated by single-pixel shifts was presented at the estimated center of the second grid stimulus. The location of the peak response to this stimulus was used as the RF center in subsequent experiments. Once the RF center was identified, the moving bar stimulus was presented in eight directions and four step-durations. The bar was $1 \times 9$ pixels. When moving in the cardinal directions, the motion spanned 9 pixels. In the diagonal directions, bar motion included more steps to cover the same distance ( 9 steps vs. 13 steps). Once the preferred direction had been estimated, bright bar (also $1 \times 9$ pixels) flashes were presented on the relevant axis. To verify full coverage of RF, this stimulus was presented over an area larger than the original motion window (at least 13 positions). In addition to these stimuli, most cells were also presented with additional stimuli following this procedure. All stimuli were presented in a pseudorandom order within stimulus blocks. All stimuli were presented three times, except for single-bar flashes, which were repeated five times. The interstimulus interval was $500 \mathrm{~ms}$ for moving stimuli and $800 \mathrm{~ms}$ for single-bar flashes (to minimize the effect of ongoing inhibition on the responses to subsequent stimuli).

Analysis. All data analysis was performed in Matlab using custom written code. Since the T4 baseline was typically stable, we included only trials in which the mean prestimulus baseline did not differ from the overall prestimulus mean for that group of stimuli by more than $10 \mathrm{mV}$. We also verified that the prestimulus mean and overall mean for that trial did not differ by more than $15 \mathrm{mV}$ (or $25 \mathrm{mV}$ for slow-moving bars, due to their strong responses). This was designed to identify those rare trials in which the trace became unstable only after the stimulus was presented. Responses were later aligned to the appearance of the bar stimulus and averaged (or the appearance of the bar in the central position in case of the eightorientation moving bar). T4 cells are expected to signal using graded synapses. Consistent with this expectation, we find that $\mathrm{T} 4$ recordings only occasionally feature very weak, fast transients $(\sim 1-2 \mathrm{mV}$ in size) that could not be verified as spikes. Therefore, we have focused our analysis on the graded (subthreshold) components of T4's responses.

Determining PD. First, eight-direction responses were aligned to the center position for each cell. Second, for the duration of bar presentation, the mean vector response was calculated for each time point as $\bar{R}(t)=\sum_{k=1}^{8} R\left(\theta_{k}, t\right) e^{i \theta_{k}}$, with $\theta_{k}$ being the direction of motion (in $45^{\circ}$ intervals) and $R\left(\theta_{k}, t\right)$ the response at that direction at time $t$. Supplementary Fig. 2 shows the result of this procedure for the normalized vector magnitude and $\bar{\theta}$. This procedure can be thought of as mimicking a downstream neuron, receiving input from T4s with the same RF center but different PDs. The $\bar{\theta}$ value for the cell was selected at the timepoint when the mean vector magnitude was maximal (repeated for four step-durations and averaged). In the polar plot of Fig. 1b, this timepoint is used for the response to each bar direction. The PD was determined as the $\theta_{k}$ value with the minimal difference to $\bar{\theta}$. For Fig. 1c, responses were then circularly shifted to align the PD with rightward motions for plotting and averaging purposes.

DSI calculation. The direction selectivity index was defined as $R(P D)-R(N D) / R(P D)$, with each response defined as the 0.995 quantile (a robust estimate of the max) within the stimulus presentation window. DSI values for the model and model variations were calculated in the same manner.

Depolarization calculation for single-position flash response. Responses were defined as the 0.995 quantile (a robust estimate of the max) of the response during the time between bar appearance and flash duration $+75 \mathrm{~ms}$. If this number did not exceed 3 s.d. of the prestimulus baseline (for all bar flashes for that cell), the response was defined as zero.

Hyperpolarization calcultion for single-position flash response. These responses were calculated as for depolarization except that the time window lasted until end of trial (due to the slower time course for inhibition) and the threshold was 2 s.d. (due to the lower magnitude of hyperpolarization). These calculations were used for Fig. $2 \mathrm{c}$ and Supplementary Fig. 3 .

Depolarization (or hyperpolarization) normalization. All detected averaged SPFRs for a given duration were normalized to the maximal depolarizing (hyperpolarizing) absolute response for that duration and that cell. Figure $2 \mathrm{c}$ shows the average of these normalized responses for a single duration. Supplementary Fig. 3a shows the sums for each cell, for four normalized depolarizing responses and the two slowest hyperpolarizing responses (since hyperpolarization was hard to detect for brief flashes). A position that showed the maximal response for all durations will, therefore, have a value of 4 for depolarizations and 2 for hyperpolarizations.

Onset-time calculation. Data during a window of $200 \mathrm{~ms}$ before stimulus presentation from all the single-bar flashes presented to a cell was used for a per-cell estimate of the s.d. of the baseline. Only presentations in which the average SPFR was detected as depolarizing were used for onset-time calculation. 
Onset time was defined as the time from stimulus presentation (after it was corrected for a small display latency) in which the response crossed $0.5 \times$ baseline s.d. This threshold value did not exceed $0.5 \mathrm{mV}$. This calculation is used in Fig. 2d,e. Because positions in the center of the RF feature a mixture of excitation and inhibition, the peak time is 'contaminated' by the inhibitory contribution and could not be used as an independent, reliable measure of the properties of the excitatory input.

Decay time calculation. The decay time (from $80 \%$ to $20 \%$ of maximal response) was calculated for all cells and all positions in which a depolarizing response was detected. If $20 \%$ of max response was not attained by the time recording ended, that data point was excluded. This calculation is used in Fig. $2 \mathrm{~d}$,e. The numbers of cells that passed the above threshold and were included in Fig. $2 \mathrm{~d}$ is, for $160 \mathrm{~ms}$ : 17, $17,17,17,17,15$, 9; for $80 \mathrm{~ms}: 15,16,17,17,16,15,6$; for $40 \mathrm{~ms}$ : 11, 13, 16, 16, 16, 10,8 ; and for $20 \mathrm{~ms}: 1,8,11,17,12,8,4$

Slope calculation (onset and decay times). To calculate the slope in Fig. 2e, onset (decay) time values from all the positions of an individual cell were fit with a linear regression. Fits were performed only when more than four positions showed responses, to account for cases in which not all positions showed detectable depolarizing responses (especially due to fast flashes).

Summed SPFRs (superposition). SPRFs were aligned to the time of the corresponding position appearance in the moving-bar stimulus. Responses were padded with zeros (since all were baseline-subtracted) to extend brief single-bar responses to the timescale of a moving bar. This procedure was used both for the two-step apparent-motion stimuli and for the moving-bar stimuli (Figs. 3 and 4 and Supplementary Figs. 4, 5 and 7). For moving-bar analysis, responses in which hyperpolarization did not return to zero by the end of the recording were padded with a linear fit to the last $250 \mathrm{~ms}$ that was extended to zero (to avoid abrupt changes). For the analysis in Fig. 4, one cell was not included because the dataset for this recording was incomplete.

Rectified SPFR sums. These were calculated as for standard sums but with all negative values in individual SPFRs set to zeros (Supplementary Fig. 5b).

Scaled center response SPFR sums. Individual SPFRs were replaced with a central response trace after rectification, scaled to the amplitude of the original positional response. This was done to eliminate the positional change in response width (Supplementary Fig. 5b).

Average traces in Fig. 4d,f and Supplementary Fig. 5a. Positions from all cells were aligned to the center zero position before averaging. All trajectories that were longer than eight positions were included in this analysis. Some were extracted from the original eight-direction stimuli (Fig. 1), which was used to determine PD, and some from additional trajectories that were presented just along the PD axis.

Squared sum in Supplementary Fig. 7. The averaged measured data (presented in Supplementary Fig. 7a) was squared (after baseline-subtraction) for both the individual presentations and the two-step positions. To generate the expected sum, individual squared responses were temporally aligned and summed.

Spatiotemporal maps in Supplementary Fig. 8. Averaged SPFRs were aligned temporally to stimulus appearance. A two-dimensional Gaussian was used to smooth the data both temporally $(\sigma=250 \mathrm{~ms})$ and spatially ( $\sigma=$ one position) using Matlab function imgaussfilt. Contour plots were generated using Matlab function contourf, with a fixed 'level list' that was manually generated to include smaller steps for the hyperpolarized range. An identical procedure was performed on the simulated SPFRs and simulated SPFRs without inhibition (see the "Conductance model" section for details).

Statistics. To determine statistically significant differences, one-sided unpaired Student's $t$ tests were used for comparing groups (Figs. 2e and 4e and Supplementary Fig. 5b). Data distribution was assumed to be normal, but this was not formally tested. No statistical methods were used to predetermine sample sizes, but our sample sizes are similar to those reported in previous publications $s^{49-51}$. Data collection and analysis were not performed blind to the conditions of the experiments.

Data-plotting conventions. All boxplots presented (except Fig. $2 \mathrm{~d}$ ) were plotted with Matlab conventions. Boxes represent the first and third quartiles, center lines represent medians, and whiskers represent the farthest point within the $\mathrm{q}_{3}+\mathrm{IQR}$ (or $\mathrm{q}_{1}-\mathrm{IQR}$ ) range. Boxplots for Fig. 2d depict only medians and quartiles due to spatial constraints. In Fig. 1, triangles denote data points outside of the plot (for 20 $\mathrm{ms}:-0.19$ and -1.14 ; for $40 \mathrm{~ms}:-0.09$ and -0.7$)$. In Fig. $4 \mathrm{e}$, triangle denotes a data point outside the plot for measured $40 \mathrm{~ms}$ : -0.7 . Most plots used the cbrewer color library from the MathWorks file exchange.

Conductance model. A model T4 neuron was implemented using the morphology of a single T4 cell, reconstructed from electron microscopy data. The neuron morphology was generously shared by K. Shinomiya and Janelia's FlyEM project team. The FlyEM team collected a dataset containing approximately half of a Drosophila optic lobe, imaged with isotropic 8-nm voxels by focused ion-beam milling scanning electron microscopy (FIB-SEM). The sample was prepared from the head of a female fly as previously reported, using high-pressure freezing followed by freeze-substituted embedding ${ }^{9,52}$. A $153 \times 85 \times 180-\mu$ m volume containing connected regions of the lamina, medulla, lobula and lobula plate was imaged. The imaged volume was segmented automatically based on an algorithm similar to one previously described ${ }^{53}$. NeuTu-EM (https://github.com/janeliaflyem/NeuTu/tree/flyem_release), in which segmented fragments of neurons are merged and split to form the complete morphology of individual neurons, was then used to proofread the segmented volume. A reconstructed T4 neuron was identified based on its distinctive morphology, with dendritic compartments spanning $\sim 20 \mu \mathrm{m}$ in the medulla and an axon projecting to a distinct layer of the Lobula Plate. The reconstructed neuron morphology contained 344 sections.

To correct for a small number of inconsistencies in the reconstructed morphology, the diameter of each dendritic section was smoothed by performing a moving average of the diameter of five adjacent sections (using the TREES toolbox ${ }^{54}$ ). The simulation was implemented and run using NEURON v.7.4 (http://www.neuron.yale.edu/neuron/). Analog synapses were placed randomly throughout the dendritic arbor (this compartment, containing 235 sections, was defined manually), and a recording electrode was attached to the soma. After identifying the primary dendritic axis (used to simulate stimulation along the PD-ND axis), we assigned each dendritic section a value corresponding to its projection on this axis ( $x^{*}$, between 0 and 1$)$. We subdivided the axis into $M=11$ intervals, where each interval contains an equal number of dendritic sections (21 sections), to map onto the 11 stimulated positions required to enclose the T4 receptive field. We used 11 intervals because the average traces in Fig. 4d, which were the reference for the simulation, spanned positions -5 to 5 (see "Analysis" subsection). Within an interval, we randomly selected a fixed number $N$ of dendritic sections $N_{\mathrm{E}}=9$ ( or $N_{\mathrm{I}}=5$ ) where excitatory (E; or inhibitory, I) graded synapses were placed. The reversal potential for excitatory synapses was set to $0 \mathrm{mV}$ and to $-70 \mathrm{mV}$ for inhibitory synapses. The resting membrane potential was set to $-65 \mathrm{mV}$. To simulate a visual input, mimicking the appearance of the bar at one position, all the synapses in the region were activated with conductance dynamics that were uniform for all $\mathrm{E}$ and different, but uniform, for all I synapses. Our model simplifies the transformation from visual input to a synaptic conductance change, so while we are not explicitly modeling the optical and neuronal prefiltering that is upstream of T4, these details are implicitly incorporated into the model, as these elements contribute to the SPFRs, which the model was fit to reproduce. To simulate an analog synapse, we used the single-electrode voltage-clamp (SEVC) point process and injected the inverse of our calculated conductance (see below) into the SEVC $R_{\mathrm{s}}$ (zero conductance corresponds to $1 \times 10^{9} \Omega$ resistance).

We computed the $\mathrm{E}$ and I conductance time course at each synapse $\left(g_{E}\left(t, x^{\star}\right)\right.$, $\left.g_{I}\left(t, x^{\star}\right)\right)$ according to the following:

$$
\begin{aligned}
\tau_{\mathrm{C}, \text { rise }} \frac{d h_{\mathrm{C}}\left(t, x^{*}\right)}{d t} & =-h_{\mathrm{C}}\left(t, x^{*}\right)+I_{\mathrm{C}}\left(t, x^{*}\right) \\
\tau_{\text {C, decay }} \frac{d f_{\mathrm{C}}\left(t, x^{*}\right)}{d t} & =-f_{\mathrm{C}}\left(t, x^{*}\right)+h_{\mathrm{C}}\left(t, x^{*}\right) \\
g_{\mathrm{C}}\left(t, x^{*}\right) & =f_{\mathrm{C}}\left(t, x^{*}\right) a_{\mathrm{C}}\left(x^{*}\right)
\end{aligned}
$$

where $\mathrm{C}=\{\mathrm{E}, \mathrm{I}\}$, and $\left(\tau_{\mathrm{C} \text {,rise }}, \tau_{\mathrm{C} \text {,decay }}\right)$ denote the rise (or decay) time constants of the synapse, $I_{C}\left(t, x^{*}\right)$ is the synaptic input, and $a_{C}\left(x^{*}\right)$ scales the conductance based on the location of the synapse on the PD-ND axis. We modeled the amplitude as a Gaussian profile

$$
a_{\mathrm{C}}\left(x^{*}\right)=A_{\mathrm{C}} e^{-\frac{\left(x^{*}-\mu_{\mathrm{C})}^{2}\right.}{2 \sigma_{\mathrm{C}}^{2}}}
$$

with an overall amplitude parameter $A_{\mathrm{C}}$, a peak location along the dendrite $\mu_{\mathrm{C}}$, and a width $\sigma_{C}$. We chose a Gaussian profile because it reasonably approximates the spatial profile of $\mathrm{E}$ and I inputs measured for the SPFRs (Fig. 2c and Supplementary Fig. 3a) and to reduce the number of parameters needed to describe the inputs to the simulated T4 neuron. The synaptic input, $I_{C}\left(t, x^{\star}\right)$, was modeled as a pulse of unit amplitude with duration $T$, where $T=20,40,80,160 \mathrm{~ms}$, and was identical for all the synapses located within one of the $M$ intervals.

Three additional parameters of the neuronal model are the membrane resistivity $\left(R_{\mathrm{m}}\right)$, membrane capacitance $\left(C_{\mathrm{m}}\right)$ and axial resistivity $\left(R_{\mathrm{a}}\right)$. For all simulations, $C_{\mathrm{m}}$ was fixed at $C_{\mathrm{m}}=1 \mu \mathrm{F} / \mathrm{cm}^{2}$. We optimized the remaining model parameters, by performing nonlinear least squares minimization between the numerical simulation results combining all stimulus positions $(M=11$ intervals) and durations ( $T$ ), resulting in 44 simulated SPFR responses, and the corresponding measured SPFRs (Supplementary Fig. 6c). The minimization used the lsqcurvefit() function from Matlab’s Optimization Toolbox. Having fit the model parameters using the single-position flash stimuli, we used the 
same optimized parameters to simulate the dynamics of the model in response to moving bars (apparent-motion stimulus). The moving-bar stimulus was implemented by sequentially activating the synaptic inputs along the dendritic axis, with each interval being active for a duration $T$.

Model manipulations. We moved all synaptic inputs ( 99 excitatory and 55 inhibitory synapses) to the first dendritic section (Fig. $5 \mathrm{~d}$ ). We then removed all conductance changes mediated by the inhibitory synapses, while keeping all other settings of the model fixed. This was accomplished by setting the duration of all inhibitory synaptic inputs to zero (Fig. 5d).

Single-compartment conductance model. We modeled the time course of the membrane potential of a T4 neuron, $V(t)$, according to the single-compartment dynamics

$$
C_{M} \frac{d V(t)}{d t}=-g_{\mathrm{L}}\left(V(t)-V_{\mathrm{L}}\right)-g_{\mathrm{E}}(t)\left(V(t)-V_{\mathrm{E}}\right)-g_{\mathrm{I}}(t)\left(V(t)-V_{\mathrm{I}}\right)
$$

where $C_{M}$ is the membrane capacitance, $g_{L}$ is the leak conductance (resulting in the integration time constant of the neuron $\left.\tau_{V}=C_{M} / g_{\mathrm{L}}\right)$, and $V_{\mathrm{L}}, V_{\mathrm{E}}$ and $V_{\mathrm{I}}$ are the resting, excitatory and inhibitory reversal potentials respectively (values in Supplementary Table 1). The excitatory and inhibitory conductances, $g_{\mathrm{C}}(t), \mathrm{C}=\{\mathrm{E}, \mathrm{I}\}$, were modeled as a Gaussian weighted linear combination of stimulus location-specific conductances, $g_{\mathrm{C}}(t)=\sum_{i=1}^{M} a_{\mathrm{C}}\left(x_{i}\right) f_{\mathrm{C}}\left(t, x_{i}\right)$ where, as for the model described in the previous section,

$$
a_{\mathrm{C}}\left(x_{i}\right)=A_{\mathrm{C}} e^{-\frac{\left(x_{i}-\mu_{\mathrm{C}}\right)^{2}}{2 \sigma_{\mathrm{C}}^{2}}}
$$

$g_{\mathrm{C}}\left(t, x_{i}\right)$, the conductance change elicited by the presentation of a stimulus $I_{\mathrm{C}}\left(t, x_{i}\right)$ in one of $M=15$ locations $x_{i}$, follows the dynamics

$$
\begin{gathered}
\tau_{\mathrm{C}, \text { rise }} \frac{d h_{\mathrm{C}}\left(t, x_{i}\right)}{d t}=-h_{\mathrm{C}}\left(t, x_{i}\right)+I_{\mathrm{C}}\left(t, x_{i}\right) \\
\tau_{\mathrm{C}, \text { decay }} \frac{d f_{\mathrm{C}}\left(t, x_{i}\right)}{d t}=-f_{\mathrm{C}}\left(t, x_{i}\right)+h_{\mathrm{C}}\left(t, x_{i}\right)
\end{gathered}
$$

Stimuli had characteristics identical to those described in the previous section. All the parameters (with the exception of the reversal potentials) were optimized to minimize the mean squared error between model responses to single-bar inputs and average T4 SPFRs.

As with our original model, solutions to the single compartment model conformed to one of the two solution clusters. The solutions either had fast $(<10 \mathrm{~ms})$ time constants for $\mathrm{E}$ and slow membrane time constants (solution cluster 1), or slower E time constants and negligible membrane time constants (solution cluster 2).

When the time constant of the neuron is negligible compared to the time scale of the stimuli and conductances $\left(\tau_{V} \rightarrow 0\right)$, the relative change of membrane potential with respect to the resting potential can be written as

$$
\Delta V(t) \equiv \frac{V(t)-V_{\mathrm{L}}}{V_{\mathrm{E}}-V_{\mathrm{L}}} \cong \frac{E(t)-\alpha I(t)}{1+E(t)+I(t)}
$$

where $\alpha=\frac{V_{\mathrm{L}}-V_{\mathrm{I}}}{V_{\mathrm{E}}-V_{\mathrm{L}}}, E(t)=g_{\mathrm{E}}(t) / g_{\mathrm{L}}$, and $I(t)=g_{\mathrm{I}}(t) / g_{\mathrm{L}}$. This is the expression used in the model schematics depicted in Fig. 6.

Note that if one combination of excitatory and inhibitory conductances elicits a hyperpolarizing response, $\Delta V_{1}=\frac{E_{1}-\alpha l_{1}}{1+E_{1}+I_{1}}<0$

while a second one produces a depolarizing response, $\Delta V_{2}=\frac{E_{2}-\alpha I_{2}}{1+E_{2}+I_{2}}>0$, under some conditions it is possible to obtain a supralinear response to the superposition of the two sets of conductances

$$
\Delta V_{\text {land } 2}=\frac{\left(E_{1}+E_{2}\right)-\alpha\left(I_{1}+I_{2}\right)}{1+\left(E_{1}+E_{2}\right)+\left(I_{1}+I_{2}\right)}>\Delta V_{1}+\Delta V_{2} .
$$

For instance, when $E_{1}=0, I_{2}=0$, supralinearity is obtained if $E_{2}>\frac{1+I_{1}-\alpha}{\alpha}$.

Life Sciences Reporting Summary. Further information on experimental design is available in the Life Sciences Reporting Summary.

Code availability. The essential code used to generate the primary results and conduct the simulations for this study are available at https://doi.org/10.25378/ janelia.c.3955843 and https://www.janelia.org/publication/simple-integration-fastexcitation-and-offset-delayed-inhibition-computes-directional.

Data availability. The data used to generate the primary results of this study are available at https://doi.org/10.25378/janelia.c.3955843 and https://www.janelia. org/publication/simple-integration-fast-excitation-and-offset-delayed-inhibitioncomputes-directional.

The split-GAL4 driver used to target T4 cells (SS02344: VT015785-p65ADZp (attP40); R42F06-ZpGdbd (attP2)) was generously provided by A. Nern (line details with expression data available from http://splitgal4.janelia.org/).

\section{References}

41. Aso, Y. et al. The neuronal architecture of the mushroom body provides a logic for associative learning. eLife 3, e04577 (2014).

42. Nern, A., Pfeiffer, B. D. \& Rubin, G. M. Optimized tools for multicolor stochastic labeling reveal diverse stereotyped cell arrangements in the fly visual system. Proc. Natl. Acad. Sci. USA 112, E2967-E2976 (2015).

43. Peng, H., Ruan, Z., Long, F., Simpson, J. H. \& Myers, E. W. V3D enables real-time $3 \mathrm{D}$ visualization and quantitative analysis of large-scale biological image data sets. Nat. Biotechnol. 28, 348-353 (2010).

44. Pfeiffer, B. D., Truman, J. W. \& Rubin, G. M. Using translational enhancers to increase transgene expression in Drosophila. Proc. Natl. Acad. Sci. USA 109, 6626-6631 (2012).

45. Demerec, M. Biology of Drosophila (Hafner Press, New York, 1965).

46. Wilson, R. I. \& Laurent, G. Role of GABAergic inhibition in shaping odor-evoked spatiotemporal patterns in the Drosophila antennal lobe. J. Neurosci. 25, 9069-9079 (2005).

47. Edelstein, A. D. et al. Advanced methods of microscope control using $\mu$ Manager software. J. Biol. Methods https://doi.org/10.14440/jbm.2014.36 (2014).

48. Reiser, M. B. \& Dickinson, M. H. A modular display system for insect behavioral neuroscience. J. Neurosci. Methods 167, 127-139 (2008).

49. Bahl, A., Serbe, E., Meier, M., Ammer, G. \& Borst, A. Neural mechanisms for Drosophila contrast vision. Neuron 88, 1240-1252 (2015).

50. Turner-Evans, D. et al. Angular velocity integration in a fly heading circuit. eLife 6, e23496 (2017).

51. Tuthill, J. C., Nern, A., Rubin, G. M. \& Reiser, M. B. Wide-field feedback neurons dynamically tune early visual processing. Neuron 82, 887-895 (2014).

52. Takemura, S. Y. et al. Synaptic circuits and their variations within different columns in the visual system of Drosophila. Proc. Natl. Acad. Sci. USA 112, 13711-13716 (2015)

53. Parag, T., Chakraborty, A., Plaza, S. \& Scheffer, L. A context-aware delayed agglomeration framework for electron microscopy segmentation. PLoS One 10, e0125825 (2015)

54. Cuntz, H., Forstner, F., Borst, A. \& Häusser, M. One rule to grow them all: a general theory of neuronal branching and its practical application. PLOS Comput. Biol. 6, e1000877 (2010). 


\section{natureresearch}

Corresponding author(s): Reiser Michael

Initial submission

Revised version

Х Final submission

\section{Life Sciences Reporting Summary}

Nature Research wishes to improve the reproducibility of the work that we publish. This form is intended for publication with all accepted life science papers and provides structure for consistency and transparency in reporting. Every life science submission will use this form; some list items might not apply to an individual manuscript, but all fields must be completed for clarity.

For further information on the points included in this form, see Reporting Life Sciences Research. For further information on Nature Research policies, including our data availability policy, see Authors \& Referees and the Editorial Policy Checklist.

\section{- Experimental design}

\section{Sample size}

Describe how sample size was determined.

\section{Data exclusions}

Describe any data exclusions.

\section{Replication}

Describe whether the experimental findings were reliably reproduced.

\section{Randomization}

Describe how samples/organisms/participants were allocated into experimental groups.

\section{Blinding}

Describe whether the investigators were blinded to group allocation during data collection and/or analysis.
We did not perform a formal sample size calculation. However, our sample size (17 recordings) exceed the standards in our field. Most reports of single-cell recordings are in the $<10$ range.

Recordings were terminated (and excluded from analysis) if response reliability diminished noticeably (as determined by intermittent current step injections or reduction in visual responses). Since the baseline potential in our recordings was typically stable, we excluded trials in which the mean pre-stimulus baseline differed from the overall pre-stimulus mean for that group of stimuli by more than $10 \mathrm{mV}$. These criteria were established after data collection, but applied uniformly on all cells.

The experimental findings were reliably reproduced, with $n=17$ recordings for most results. Individual examples as well as population means are presented.

Within each stimulus set, the stimuli were presented in a pseudo-random manner (e.g. single position bar flashes were presented with positions and durations randomly interleaved).

Blinding was not possible for this study since there is just one experimental group. 


\section{Statistical parameters}

For all figures and tables that use statistical methods, confirm that the following items are present in relevant figure legends (or in the Methods section if additional space is needed).

$\mathrm{n} / \mathrm{a} \mid$ Confirmed

The exact sample size ( $n$ ) for each experimental group/condition, given as a discrete number and unit of measurement (animals, litters, cultures, etc.)

A description of how samples were collected, noting whether measurements were taken from distinct samples or whether the same sample was measured repeatedly

A statement indicating how many times each experiment was replicated

The statistical test(s) used and whether they are one- or two-sided (note: only common tests should be described solely by name; more complex techniques should be described in the Methods section)

$\searrow$ A description of any assumptions or corrections, such as an adjustment for multiple comparisons

$\bigotimes$ The test results (e.g. $P$ values) given as exact values whenever possible and with confidence intervals noted

$\bigotimes$ A clear description of statistics including central tendency (e.g. median, mean) and variation (e.g. standard deviation, interquartile range)

Clearly defined error bars

See the web collection on statistics for biologists for further resources and guidance.

\section{- Software}

Policy information about availability of computer code

\section{Software}

Describe the software used to analyze the data in this study.

Data were analyzed using custom code written in Matlab 2015a.

Multicompartment conductance model was implemented in NEURON v7.4. Data acquisition and control of LED arena was implemented using LabVIEW 2013 (v.13.0.1f2).

For manuscripts utilizing custom algorithms or software that are central to the paper but not yet described in the published literature, software must be made available to editors and reviewers upon request. We strongly encourage code deposition in a community repository (e.g. GitHub). Nature Methods guidance for providing algorithms and software for publication provides further information on this topic.

\section{- Materials and reagents}

Policy information about availability of materials

\section{Materials availability}

Indicate whether there are restrictions on availability of unique materials or if these materials are only available for distribution by a for-profit company.

\section{Antibodies}

Describe the antibodies used and how they were validated for use in the system under study (i.e. assay and species).

\section{Eukaryotic cell lines}

a. State the source of each eukaryotic cell line used.

b. Describe the method of cell line authentication used.

c. Report whether the cell lines were tested for mycoplasma contamination.

d. If any of the cell lines used are listed in the database of commonly misidentified cell lines maintained by ICLAC, provide a scientific rationale for their use.
No restrictions on availability

Antibodies used to verify expression in T4 neurons have been widely validated in Drosophila neuroscience studies, for example Aso, Y. et al. eLife (2014).

Rat $\alpha$-FLAG Tag (DYKDDDDK Epitope Tag). Novus Biologicals. \# NBP1-06712 nc82 - Mouse $\alpha$-bruchpilot. Developmental Studies Hybridoma Bank. \#nc82-s

N/A

N/A

N/A 
Policy information about studies involving animals; when reporting animal research, follow the ARRIVE guidelines

11. Description of research animals

Provide details on animals and/or animal-derived materials used in the study.

female Drosophila melanogaster pJFRC28-10XUAS-IVS-GFP-p104 in attP2 crossed to stable split-GAL4 SS02344 ( VT015785-p65ADZp (attP40); R42F06-ZpGdbd (attP2))

Policy information about studies involving human research participants

\section{Description of human research participants}

Describe the covariate-relevant population

characteristics of the human research participants. 